\title{
On the use of Stochastic Dynamic Programming for Evaluating a Power-Split CVT in a Wheel Loader
}

\author{
Tomas Nilsson \\ Department of \\ Electrical Engineering \\ Linköping University \\ Linköping, Sweden \\ Email: tnilsson@isy.liu.se
}

\author{
Anders Fröberg \\ Volvo CE \\ Eskilstuna, Sweden \\ Email: anders.froberg@volvo.com
}

\author{
Jan Åslund \\ Department of \\ Electrical Engineering \\ Linköping University \\ Linköping, Sweden \\ Email: jaasl@isy.liu.se
}

\begin{abstract}
Complex transmission concepts may enable high fuel efficiency but require much effort in controller development. This effort should only be spent if the potential of the concept if high, a potential which can be determined using optimization techniques. This paper examine the use of stochastic dynamic programming for transmission potential evaluation, applied on a wheel loader. The concepts evaluated is the present automatic gearbox and a multi-mode CVT (MM-CVT). A probabilistic driving cycle is created from a measurement including 34 loading cycles. Trajectory optimization is performed both against probabilistic and deterministic cycles. The paper shows that the introduction of a probabilistic load highly affect the application of optimization. It is also shown that the MM-CVT has approximately $20 \%$ lower minimum fuel requirement than the present transmission, and that this number is not sensitive to the quality of the prediction.
\end{abstract}

\section{INTRODUCTION}

\section{A. Wheel loader transmissions}

Wheel loader operation is often highly transient and contain episodes of low speed and high tractive effort. The present transmission is well suited for this operation, but in some modes of operation the efficiency is low. In this transmission both operational advantages and efficiency drawbacks are highly related to the use of a torque converter. One alternative to the present setup is infinitely variable transmissions, such as hydrostatic [1] or diesel-electric [2] transmissions. The drawback with this type of transmission is that the repeated power conversions reduce the efficiency. Power-split constructions has two or more parallel power paths [3], [4], allowing for increased effciency. Multi-mode CVTs are constructed so that several power-split layouts can be realized with the same device, enabling high efficiency at widely spaced gear ratios. In this paper, just as in [5], a hydrostatic multi-mode CVT (MM-CVT) concept is analysed.

\section{B. Dynamic optimization}

The successful implementation of the conceptual MM-CVT transmission depends on the quality of the controller. But since controller development is far from trivial, see for example the work in [6], time and effort should not be spent developing controllers unless the potential of the concept is high. The potential of mechanical or control concepts can be determined by trajectory optimization [7]-[9]. Such optimization rely on a prediction, deterministic or probabilistic, of future load. Since wheel loaders are off-road vehicles with highly transient operating patterns, accurate prediction is not readily available. This paper examine the use of stochastic dynamic programming for evaluating the fuel usage minimization potential of the present drivetrain and of the MM-CVT concept.

\section{PROBLEM}

\section{A. Problem statement}

The problem studied in this paper is the minimization of the total amount of fuel consumed during a pre-specified driving mission. Deterministic and probabilistic load cases, based on statistics from measurements, are used. No deviation from the load case is allowed and therefore it is assumed, just as in [8], [10], to be known beforehand.

The system consist of an engine connected to a working hydraulics pump and the transmission, according to Figure 1. Two transmission concepts are compared; the present torque converter/automatic gearbox and a multi-mode CVT (MMCVT). Models of similar complexity are used in both cases for a fair comparison. Identical diesel engine and hydraulic pump models are used in the two concepts.

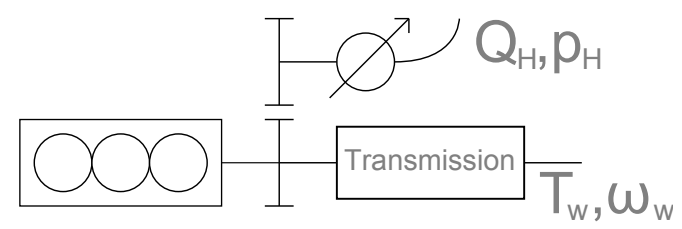

Fig. 1. General drivetrain setup

\section{B. Load cases}

The load cases are specified by the wheel speed $\omega_{w}$, wheel torque $T_{w}$, hydraulic pressure $p_{H}$ and hydraulic flow $Q_{H}$. In the deterministic cases all load components are deterministic. In the stochastic case $p_{H}$ and $Q_{H}$ are stochastic, but since rapidly varying vehicle speed is unrealistic, $\omega_{w}$ is deterministic, which also affect $T_{w}$. The wheel torque is divided into two parts; $T_{w}=T_{A}\left(\frac{d \omega_{w}}{d t}\right)+T_{D}$ where $T_{A}$ depend on the vehicle 
acceleration and is deterministic, while $T_{D}$ include force on the bucket and rolling resistance and is stochastic.

Wheel loader usage can typically be characterized as loading cycles [11]. A measurement sequence with 34 such cycles has been used as basis for the stochastic load cases. When creating the stochastic cases the cycles were adjusted so that the direction changes occur at the exact same times in each cycle. At each time instant the mean $E$ and standard deviation $\sigma$ of each of the stochastic parts of the load is taken. The load cycle consists of the three alternatives $\left(W_{Y}=[E-\sigma, E, E+\right.$ $\sigma]$ ) of each of the three independent stochastic components $Y \in\left[T_{D}, p_{h}, Q_{h}\right]$. Each of the alternative loads also has a corresponding probability $P\left(W_{Y}\right)$. The probabilities are assumed to be independent $P\left(W_{Y}\left(t_{k}\right) \mid W_{Y}\left(t_{k-1}\right)\right)=P\left(W_{Y}\left(t_{k}\right)\right)$. The stochastic cycle is labeled the 'SDP' cycle. The mean cycle is used as a deterministic case, and labeled the 'DDP' cycle.

\section{Engine model}

The engine speed $\omega_{e}$ dynamics is modeled as an inertia $I_{e}$ which is affected by the engine torque $T_{e}$, the transmission torque $T_{T}$ and the hydraulic pump torque $T_{H}$.

$$
\frac{d \omega_{e}}{d t} \cdot I_{e}=T_{e}-T_{T}-T_{H}
$$

The relation between injected fuel and engine torque is described by a quadratic Willan's efficiency model [12]

$$
T_{e}=e \cdot \frac{q_{l h v} n_{c y l}}{2 \pi n_{r}} \cdot m_{f}-T_{l o s s}
$$

in which $q_{l h v}$ is the lower heating value, $n_{c y l}$ is the number of cylinders, $n_{r}$ is the number of strokes per injection, $m_{f}$ is fuel mass per injection and

$$
\begin{aligned}
e & =e_{0}-e_{1} m_{f} \\
e_{0} & =e_{00}+e_{01} \omega_{e}+e_{02} \omega_{e}^{2} \\
e_{1} & =e_{10}+e_{11} \omega_{e} \\
T_{\text {loss }} & =T_{L 0}+T_{L 2} \omega_{e}^{2}
\end{aligned}
$$

in which $e_{00}, e_{01}, e_{02}, e_{10}, e_{11}, T_{L 0}, T_{L 2}$ are Willan's parameters which have been fitted against a $260 \mathrm{~kW}$ diesel engine. Figure 2 presents the efficiency map of this engine. The gray lines indicate allowed operating region (minimum speed and maximum torque) and the black line indicates the static optimal operating points for each output power. The figure also show efficiency levels and output power lines with $k W$ markings.

\section{Reference transmission model}

The transmission of the reference vehicle consist of a torque converter and a four speed forward/reverse automatic gearbox. The main source of losses in this transmission is the torque converter, which is modeled according to Equation (4).

$$
\begin{aligned}
& \nu=\frac{\omega_{t}}{\omega_{p}} \\
& T_{p}=M_{P}(\nu)\left(\frac{\omega_{p}}{\omega_{\text {ref }}}\right)^{2} \\
& T_{t}=\mu(\nu) T_{p}
\end{aligned}
$$

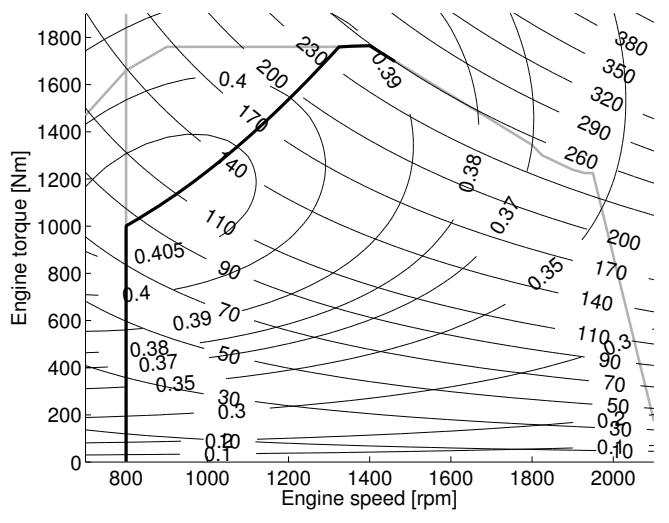

Fig. 2. Engine map with efficiency curves, output power with $k W$ markings, minimum speed and maximum torque bounds and the static optimal line

in which index- $p$ is engine side and index- $t$ is gearbox side. $M_{P}(\nu)$ and $\mu(\nu)$ are maps measured at $\omega_{\text {ref }}$.

\section{E. Multi-mode CVT (MM-CVT) model}

The concept transmission is a three mode $\left(m_{T} \in[1,2,3]\right)$ CVT with a structure similar to devices used in [5], [13]. The CVT functionality is provided by two hydraulic machines which together form a 'variator'. Changing gear ratio within a mode is done by altering the ratio between the displacement of the hydraulic machines. Mode shifts are performed at the extremals of the variator displacement by applying and releasing clutches, and mode shifts at these points do not change the total gear ratio for an ideal transmission. The main source of losses in this concept is the variator, which is modeled according to Equations (5) and (6). This model is based on [14].

$$
\begin{aligned}
& \varepsilon_{1} D_{v} \omega_{1} \pm p_{v}\left(2 C_{l a}+\left(\omega_{1}+\omega_{2}\right) C_{l b}\right)-\varepsilon_{2} D_{v} \omega_{2}=C_{v T} \dot{p_{v}} \\
& \varepsilon_{n} D_{v} p_{v}-T_{n} \pm\left(C_{l c} \omega_{n}+C_{l d} p_{v}\right)=0
\end{aligned}
$$

The index $n=1,2$ denote the two machines, $D_{v}$ is maximum displacement, $\varepsilon_{n} \in(0,1)$ is relative displacement, $\omega_{n}$ is axle speed, $p_{v}$ is variator hydraulic pressure, $T_{n}$ is torque and $C_{l}$ are efficiency parameters. The sign in the equations depend on the power flow direction. Equation (5) describes hydraulic flow and Equation (6) describes torque at each machine. The variator is constructed so that $\varepsilon_{1}+\varepsilon_{2}=1$. The $p_{v}$ dynamics is assumed to be fast compared to the engine dynamics, so that the time constant $C_{v T}$ can be assumed to be zero. At mode shifts the speed differences over the involved clutches are close to zero, so the clutch losses are small.

\section{F. Hydraulics model}

Working hydraulics pressure and flow are supplied by a variable displacement pump connected to the engine axle. Equations (7) and (8) describe this pump.

$$
\begin{aligned}
& Q_{H}=\varepsilon_{H} D_{H} \omega_{e} \\
& Q_{H} p_{H}=\eta_{H} T_{H} \omega_{e}
\end{aligned}
$$



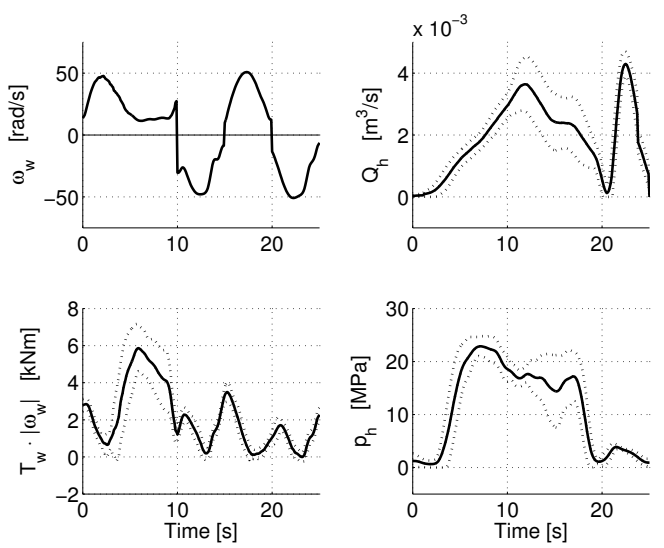

Fig. 3. Deterministic and stochastic load cases

in which $D_{H}$ is maximum displacement, $\varepsilon_{H} \in[0,1]$ is relative displacement and $\eta_{H}$ is pump efficiency.

\section{METHOD}

\section{A. Load cases}

Wheel loader usage is usually described in a 'cycle' framework. For convenient data analysis an automatic loading cycle identifier has been developed. This detects events such as bucket filling and driving direction change, the event sequence is then searched for patterns, described in an automata language [15], corresponding to loading cycles. In this work a measured sequence of 34 short loading cycles with durations between $21.5 \mathrm{~s}$ and $30.6 \mathrm{~s}$ is used. The time scales have been altered into $5 \mathrm{~s}$ forward towards bucket filling, $5 \mathrm{~s}$ bucket filling, $5 s$ reversing, $5 s$ forward towards, and including, bucket emptying and finally $5 s$ reversing.

Figure 3 shows the load cases used. The continuous lines shows the load that was actually applied. The dotted lines are the alternative loads, which along with the actual load were used as possible future loads in the stochastic cycle. The actual load was also used as the deterministic load case.

\section{B. Dynamic programming}

Denote the applied load $w$ and the discretized state $x \in X$, controls $u \in U$ and the time $t_{k}$ with $k=0,1, \ldots, N$. The optimization problem can then be stated as

$$
\min _{u \in U}\left(J_{N}\left(x_{N}\right)+\sum_{k=0}^{N-1} g\left(x_{k}, u_{k}, w_{k}\right)\right)
$$

The deterministic dynamic programming (DDP) algorithm that recursively solves this problem can, according to [16] and [17], be formulated as

$$
J_{k}\left(x_{k}\right)=\min _{u \in U}\left(g\left(x_{k}, u_{k}, w_{k}\right)+\tilde{J}_{k+1}\left(x_{k+1}\left(x_{k}, u_{k}, w_{k}\right)\right)\right)
$$

in which $\tilde{J}_{k+1}$ is found by interpolating over $J_{k+1}$. The algorithm, as applied here, is described in detail in [10]. In stochastic dynamic programming (SDP) there is some uncertainty in $w_{k}$. The algorithm is then expressed as the minimization of the expected value

$$
J_{k}\left(x_{k}\right)=\min _{u \in U} E\left[g\left(x_{k}, u_{k}, w_{k}\right)+\tilde{J}_{k+1}\left(x_{k+1}\left(x_{k}, u_{k}, w_{k}\right)\right)\right]
$$

Here the load is described by $w_{k} \in W_{k}$ along with the corresponding probability. Then

$$
\begin{aligned}
& E\left[g\left(x_{k}, u_{k}, w_{k}\right)+\tilde{J}_{k+1}\left(x_{k+1}\left(x_{k}, u_{k}, w_{k}\right)\right)\right]= \\
& \sum_{w_{m} \in W_{k}} p_{m}\left[g\left(x_{k}, u_{k}, w_{m}\right)+\tilde{J}_{k+1}\left(x_{k+1}\left(x_{k}, u_{k}, w_{m}\right)\right)\right]
\end{aligned}
$$

Further details about SDP can be found in [18]. This method is well known, does not require initial guesses and guarantees global optimum. The drawback is that the number of simulations and interpolations grows rapidly with the number of states and controls.

Regardless of transmission, the only dynamics of this vehicle is in the engine speed (Equation (1)). This makes it natural to use the engine speed as a state in the optimization. In both transmission setups there are some issues, which are described in the following parts, regarding this choice.

1) Application to the reference vehicle: The output torque from the torque converter depends directly on the input/output speed ratio. The engine speed is therefore implicitly given by the transmission speed and load. This also means that a rapidly varying load requires a rapidly varying engine speed, while the engine inertia on the other hand prevents rapid speed changes. This conflict can be solved in at least two different ways. Either the the engine inertia is assumed to be zero, or the load is smoothened by adding a brake torque to the output torque. Assuming zero inertia, and not using the egine speed as a state, will inevitably lead to non-physical speed changes if there are rapid output torque changes. Not only does this mean that the engine will always be able to operate at the best possible operating point for the corresponding load, but it also means that little or no considaretion has to be made to uncertainties in future load. If the inertia is not assumed to be zero, and the engine speed is a state, the transmission can not provide arbitrarily fast changes in output torque. The engine speed needs to be equal to or higher than that given by the highest possible output speed and torque, whether certain or probabilistic, at all times. There will therefore in general be excess output torque, which has to be absorbed by the brakes as a loss. Figure 4 illustrates the resulting brake history for the deterministic and stochastic loads. The following analysis of this work includes both the $I_{e}=0$ and the $I_{e} \neq 0$ setups.

Regardless of whether the engine speed is a state of the model or not, the minimum engine speed cause a lower limit on the output torque at low vehicle speeds. If the transmission load is less than what is given by the minimum engine speed and acceleration is not desired, a brake torque has to be applied. Therefore both setups require the control signals injected fuel $m_{f}$, brake torque $T_{b}$ and gear change $\Delta g_{T}$, along 

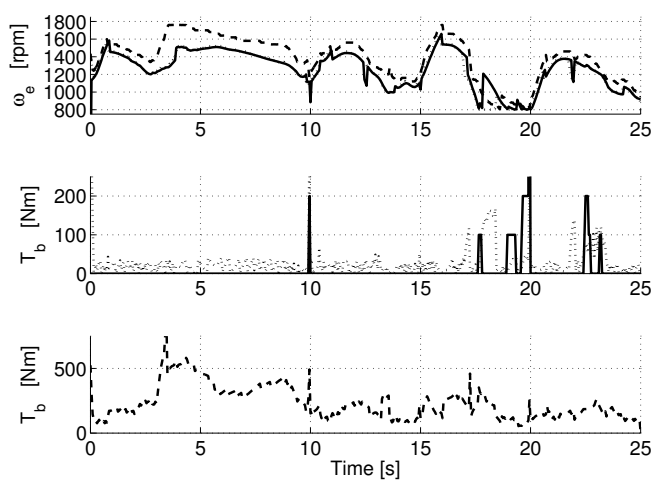

Fig. 4. Engine speed and brake torque for the reference vehicle. The continuous lines is the deterministic $I_{e}=0$ solution, the dotted is the deterministic $I_{e} \neq 0$ solution and the dashed is the stochastic $I_{e} \neq 0$ solution

with gear $g_{T}$ as a state. In the setup with engine inertia $I_{e} \neq 0$, the engine speed $\omega_{e}$ is also a state.

2) Application to the MM-CVT vehicle: Since $\omega_{e}(\varepsilon)$ is always invertible for this concept either $\omega_{e}$ or $\varepsilon$, along with $m_{T}$, can be used as state. Since the speed will increase for one of the hydraulic machines when $\varepsilon$ gets close to 0 or 1 , the losses increase in these regions. Therefore it is desirable to have high state grid density near the extremes of $\varepsilon$, which implies using $\varepsilon$ as state. The possibility of restrictions on $\Delta m_{T}$, especially during mode shifts, also points toward using $\varepsilon$ as state. Since the dynamics are described in terms of $\omega_{e}$ this would imply the following computational scheme:

$$
\varepsilon_{k} \stackrel{W_{k}}{\longrightarrow} \omega_{e, k} \stackrel{\frac{d \omega_{e}}{d t}}{\longrightarrow} \omega_{e, k+1} \stackrel{W_{\kappa}}{\longrightarrow} \varepsilon_{k+1}
$$

In the first and last steps the load is required, since $\omega_{e}(\varepsilon)$ depends on the load. At the last step a choice has to be made whether to use $\kappa=k$ or $\kappa=k+1$. Using $\kappa=k$ is equivalent to making a variable change in Equation (1) from $\frac{d \omega_{e}}{d t}$ to $\frac{d \varepsilon}{d t}$. This choice of $\kappa$ does not guarantee continuity in $\omega_{e}$, which makes it possible for the optimizer to draw a net power from the engine inertia. This is illustrated by Figure 5 which displays $\omega_{e, k}$ and $\omega_{e, k+1}$ from the solution to the stochastic load case. These lines should coincide for the engine speed to be continuous. $\kappa=k+1$ on the other hand guarantees continuous $\omega_{e}$ and works well for a deterministic load, but in the stochastic case this causes a quadratic increase in load combinations, since $\varepsilon_{k+1}$ would have to be calculated for all combinations of $W_{k}, W_{k+1}$. This would cause an unacceptable increase in calculation time. This means that for SDP it is not practical to use $\varepsilon$ as a state, and this is not included further in this work, instead $\omega_{e}$ is used as a state. $\omega_{e}\left(\varepsilon, m_{T}\right)$ may only be non-invertible in small regions near $\varepsilon=\{0,1\}$, so instead of using $m_{T}$ as a state, the $m_{T}$ which give highest efficiency is used in ambiguous cases. Therefore in this model the state is engine speed $\omega_{e}$ and the control is injected fuel $m_{f}$.
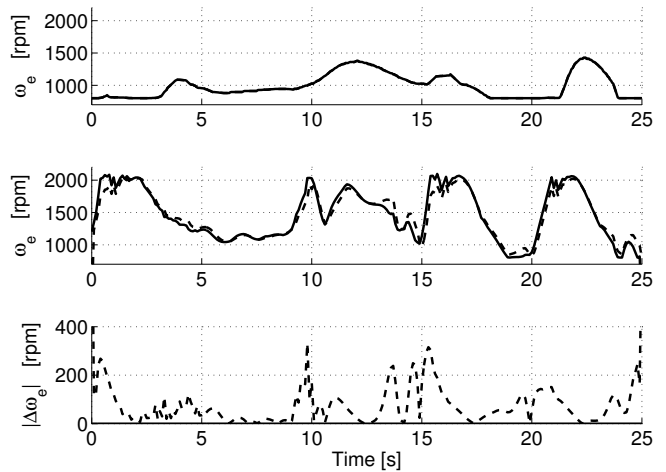

Fig. 5. The top two figures show the engine speeds $\omega_{e, k}$ (continuous) and $\omega_{e, k+1}$ (dotted) for $\varepsilon, m_{T}$ (top) and $\omega_{e}$ (middle) as state. The bottom figure shows the speed errors for the two alternatives

\section{RESULTS}

Table I summarizes the fuel usage with stochastic and deterministic loads for the three setups that have been analyzed. The load cases are the deterministic and stochastic cases presented in Figure 3. The MM-CVT fuel saving potential in the deterministic case is about $20 \%$. In the stochastic case the potential is between $20 \%$ and $35 \%$, depending on the reference vehicle model.

TABLE I

REFERENCE AND MM-CVT VEHICLE FUEL USAGE

\begin{tabular}{c|cccc} 
& MM-CVT & Ref. $\left(I_{e}=0\right)$ & Ref. $\left(I_{e} \neq 0\right)$ & Saving [\%] \\
\hline DDP & 155 & 196 & 199 & $19.8-20.9$ \\
SDP & 163 & 200 & 256 & $18.0-36.2$
\end{tabular}

Figure 6 compares the SDP solutions for the two choices of states for the reference vehicle. The top figure shows the engine speed, which for the $I_{e} \neq 0$ vehicle makes a few nonphysical jumps. The engine speed of the $I_{e}=0$ vehicle is always above that which corresponds to the highest possble hydraulic flow, while the $I_{e} \neq 0$ vehicle engine speed may drop below this since there is not an infinite cost directly associated to this. The middle figure shows the brake torque, which is close to zero most of the time for the $I_{e}=0$ vehicle while there, as expected, is constant braking for the $I_{e} \neq 0$ vehicle. The bottom figure shows that the $I_{e} \neq 0$ makes more frequent gear changes. This may be because the cost associated with gear changes is relatively lower for this vehicle.

Figure 7 illustrates the SDP solution for the MM-CVT vehicle. The top figure shows the engine speed, which is always above that which corresponds to the highest possble hydraulic flow since there is an infinite cost in the cost-to-go map directly associated to lower speeds. It can be seen that the engine speed is in general close to the lowest possible at each instant. The middle figure shows the variator displacement ratio andwhile the bottom figure shows the CVT mode.

Figure 8 shows the torque converter input and output speed (top), torque (middle) and power (bottom) for the $I_{e}=0$ reference vehicle in the SDP load case. This figure indicates the direct relation between the speed difference and the input 

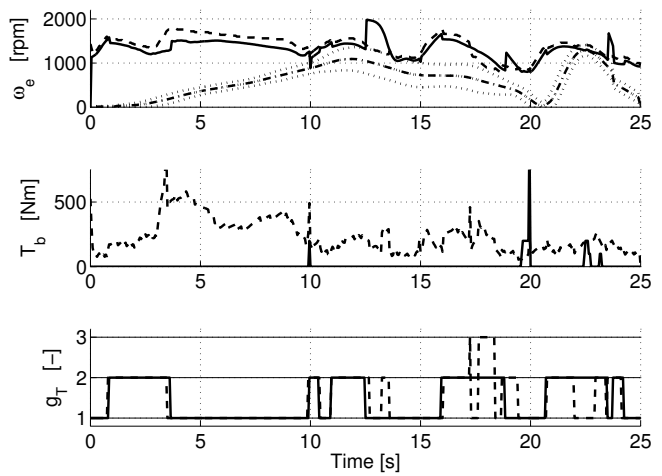

Fig. 6. Engine speed $\omega_{e}$, brake torque $T_{b}$ and gear $g_{T}$ used for the $I_{e}=0$ (continuous) and $I_{e} \neq 0$ (dashed) reference vehicle in the stochstic load case. The dotted lines show the minimum engine speeds given by the three hydraulic flow alternatives
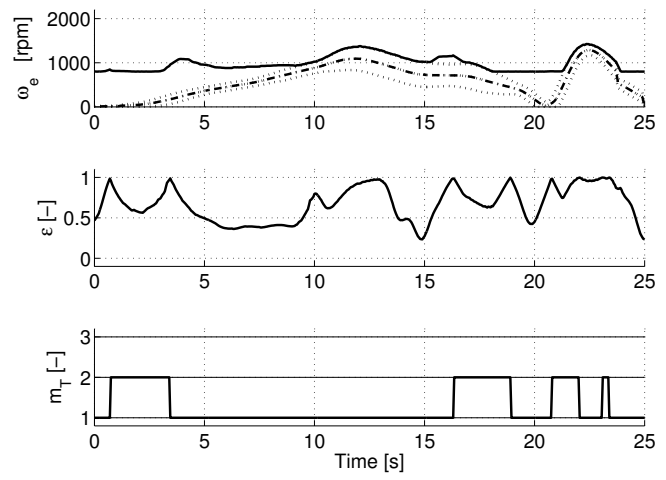

Fig. 7. Engine speed $\omega_{e}$, variator dispalcement ratio $\varepsilon$ and CVT mode $m_{T}$ used for the MM-CVT vehicle in the stochstic load case

and output torques. The bottom figure shows that the main torque converter loss is at $4 s-9 s$ during which time the bucket is beeing filled. At the driving direction change at $15 \mathrm{~s}$ there efficiency is very low, but due to the short time of this episode the energy loss is relatively small.
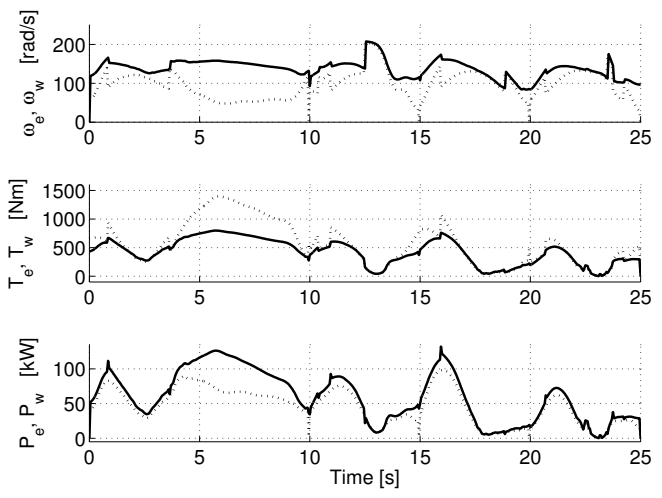

Fig. 8. Torque converter speed, torque and power input (continuous) and output (dashed) for the reference vehicle in the stochstic load case

Figure 9 shows the transmission input and output speed (top), torque (middle) and power (bottom) for the MM-CVT vehicle in the SDP load case. This figure indicates the high efficiency at a wide gear ratio range of this transmission, especially when compared to the operation of the reference transmission, as displayed in Figure 8.


Fig. 9. Transmission speed, torque and power input (continuous) and output (dashed) for the MM-CVT vehicle in the stochastic load case

Figure 10 shows the efficiencies and losses of the $I_{e}=0$ reference vehicle and the MM-CVT vehicle, divided into engine, transmission and hydraulic pump in the stochastic load case. This shows that while the efficiency of the transmission is greatly improved when switching to the MM-CVT transmission, the largest energy loss reduction is caused by a better choice of engine operating point. It should be noted that this is despite the reference vehicle engine having zero inertia, thus beeing able to instantly change operating point.
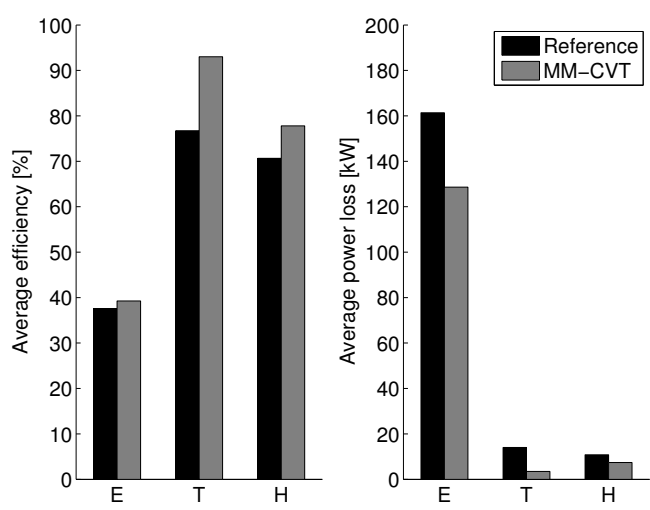

Fig. 10. Comparison of efficiencies (left) and losses (right) divided into engine (E), transmission (T) and hydraulic pump $(\mathrm{H})$, for the $I_{e}=0$ reference vehicle and the MM-CVT vehicle in the stochstic load case

Figure 11 compares the operation of the reference vehicle in the deterministic and stochastic load cases. The figure shows that at some instances in the deterministic case a higher gear can be used, causing a lower engine speed. This is acompanied by more frequent but less powerful braking.

Figure 12 compares the operation of the MM-CVT vehicle in the deterministic and stochastic load cases. The figure shows that the CVT mode is always higher and the engine speed is always lower in the deterministic case. The difference is more pronounced for this vehicle than for the reference, as shown 



Fig. 11. Comparison of engine speed $\omega_{e}$, engine torque $T_{e}$, gear $g_{T}$ used and brake torque $T_{b}$ for the $I_{e}=0$ reference vehicle with deterministic (dotted) and stochastic (continuous) loads

in Figure 11, since that vehicle has less freedom for choice of engine speed and do not have this speed as state.


Fig. 12. Comparison of engine speed $\omega_{e}$, engine torque $T_{e}, \mathrm{CVT}$ mode $m_{T}$ and variator displacement ratio $\varepsilon$ for the MM-CVT vehicle with deterministic (dotted) and stochastic (continuous) loads

\section{SUMMARY AND CONCLUSIONS}

This paper uses deterministic and stochastic dynamic programming for comparing the minimum fuel potential of two transmission concepts; the present solution with a torque converter and an automatic gearbox, and a multi-mode CVT concept. The analysis is made both for evaluating the potential benefit in fuel consumption saving potential of altering the transmission, and for evaluating the use of stochastic dynamic programming for making this analysis less sensitive to prediction uncertainties.

It is shown that the feasible choice of states in the optimization is highly affected by the change from a deterministic to a stochastic load. For the reference vehicle the engine speed cannot be used as state, since the output torque is directly dependent on the engine speed. A stochastic output torque therefore necessitates high engine speed, which cause an execess output torque which has to be balanced by the brakes, producing losses. Instead the engine inertia is assumed to be zero, and the engine speed is calculated from the transmission output speed and torque. For the MM-CVT vehicle the variator displacement ratio cannot be used as state, since this would not guarantee continuous engine speed, and the optimizer may therefore draw a net energy from the engine inertia.

It is also shown that the MM-CVT concept has a minimum fuel consumption which is about $20 \%$ lower than that of the present torque converter and automatic gearbox transmission.

\section{REFERENCES}

[1] K.-E. Rydberg, "Hydrostatic drives in heavy mobile machinery - new concepts and development trends," in International Off-Highway \& Powerplant Congress \& Exposition. SAE, 1998.

[2] R. Filla, "Alternative systems solutions for wheel loaders and other construction equipment," in 1st CTI Forum Alternative, Electric and Hybrid Drive Trains. CTI, 2008.

[3] B. Carl, M. IIvantysynova, and K. Williams, "Comparison of operational characteristics in power split continuously variable transmissions," in Comercial Vehicle Engineering Congress and Exhibition. SAE, 2006.

[4] S. Gramattico, A. Balluchi, and E. Cosoli, "A series-parallel hybrid electric powertrain for industrial vehicles," in 2010 IEEE Vehicle Power and Propulsion Conference. IEEE, 2010, pp. 1-6.

[5] S. Savaresi, F. Taroni, F. Previdi, and S. Bittanti, "Control system design on a power-split cvt for high-power agricultural tractors," IEEE/ASME Transactions on Mechatronics, vol. 9, no. 3, pp. 569-579, 2004.

[6] R. Zhang, "Multivariable robust control of nonlinear systems with application to an electro-hydraulic powertrain," Ph.D. dissertation, University of Illinois, 2002.

[7] G. Paganelli, T. Guerra, S. Delprat, J. Santin, M. Delhom, and E. Combes, "Simulation and assessment of power control strategies for a parallel hybrid car," Proceedings of the Institution of Mechanical Engineers, part D: Journal of Automobile Engineering, vol. 214, pp. 705-717, 2000.

[8] R. Pfiffner, "Optimal operation of cvt-based powertrains," Ph.D. dissertation, ETH, Zurich, 2001.

[9] A. Sciarretta and L. Guzzella, "Control of hybrid electric vehicles," Control Systems, IEEE, vol. 27, pp. 60-70, 2007.

[10] T. Nilsson, A. Fröberg, and J. Åslund, "Optimized engine transients," in 7th IEEE Vehicle Power and Propulsion Conference. IEEE, 2011.

[11] W. Fengyuan, Z. Jian, S. Ruifeng, and Y. Fei, "Analysis on the performance of wheel loades in typical work cycle," Applied Mechanics and Materials, vol. 148, pp. 526-529, 2012.

[12] G. Rizzoni, L. Guzzella, and B. Baumann, "Unified modeling of hybrid electric vehicle drivetrains," IEEE/ASME Transactions on Mechatronics, vol. 4, pp. 246-257, 1999.

[13] M. Tanelli, F. Codeca, S. Savaresi, F. Taroni, and F. Previdi, "On transmission-ratio computation for the control of a continuously variable transmission in agricultural tractors," in Proceedings of the 2007 American Control Conference. IEEE, 2007, pp. 5730-5735.

[14] J. Lennevi, "Hydrostatic transmission control, design methodology for vehicular drivetrain applications," Ph.D. dissertation, Linköping University, 1995.

[15] D. Kelley, Automata and Formal Languages. Prentice Hall, 1998.

[16] R. Bellman, Dynamic Programming. Princeton University Press, 1957.

[17] D. Bertsekas, Dynamic Programming and Optimal Control, 3rd ed. Athena Scientific, 2005, vol. 1.

[18] S. Ross, Introduction to Stochastic Dynamic Programming. Academic Press, 1983. 